\title{
An Exploration of Pregnant Women Smokers Knowledge of Accessing 'Stop Smoking' Services in Wales
}

\author{
Marian Olamide Owoniyi ${ }^{1}$, Dr. Olutoyin Elizabeth Okeya ${ }^{2}$ \\ ${ }^{1}$ Cardiff and Vale University Health Board, Cardiff, Wales. \\ ${ }^{2}$ Edge Hill University, Faculty of Health, Social Care \& Medicine, Ormskirk, Lancashire. England.
}

\begin{abstract}
The problem of smoking in pregnancy has remained a challenge to both public health professionals and Welsh Government with the low uptake of stop smoking services by pregnant women. Despite the evidence base for stop smoking, services have neither been implemented consistently nor robustly across Wales. Hence the need to develop a service improvement project like 'Models for Access to Maternal Stop Smoking Support' (MAMSS) to provide new ways of supporting pregnant women who smoke, alongside the current national Stop Smoking Services.
\end{abstract}

The study explored the experiences and views of pregnant women smokers accessing stop smoking services in Wales.

The need for suitable training was reported amongst public health professionals involved in providing stop smoking service for pregnant women. Most women wanted to reduce and not stop smoking; also available opportunities were important in determining the ability to access and deliver services, with the use of carbon monoxide (CO) monitors positively influencing the receptiveness of the pregnant women. Midwives were however reluctant to create an image of enforcing stop smoking and a holistic approach was advocated by some staff members to encourage health education and promotion. Overall, a specialist service such as that provided by the MAMSS project was viewed as appropriate.

Public health professionals understood their roles and the advantages of the $\mathrm{CO}$ monitors in encouraging quitting/stop smoking in pregnancy. Specialist midwives made positive impacts on the pregnant smoker's receptiveness to stop smoking support. Both staff and pregnant women acknowledged that accessibility and flexibility of service were key determinants of service delivery and service uptake, whilst incorporating an approach that is supportive rather than enforcing. This electronic document is a "live" template and already defines the components of your paper [title, text, heads, etc.] in its style sheet.

Keywords:- Stop Smoking, Public Health, Carbon Monoxide, Midwives and Pregnant Women.

\section{INTRODUCTION}

In Wales, the NHS-SSS known as Stop Smoking Wales (SSW) is a specialist service for adults wanting help to give up smoking. It is funded by the Welsh Government and offers a 6-8-week treatment programme based on the withdrawal oriented treatment model (James, 2007). The programme provides; advice on successful strategies for quitting, signposting to information on pharmacological aids, encouragement, support and guidance throughout the quitting process, help to set a quit date, follow-up at 4 weeks \& 12 months, and relapse prevention sessions (James, 2007). Stop Smoking Wales delivers SSS to its pregnant clients using an evidence-based approach called the Maudsley Model which focuses on nicotine-dependent smokers, through group or one-to-one sessions using behavioural therapy and Nicotine Replacement Therapy (NRT) (Bauld, 2010). Members of staff involved with pregnant smokers who are neither part of SSW nor part of a specialist service are guided by the NICE published guidance of Public Health Guidance 26 (PH26). This was tailored to the pregnant woman in a sensitive way with the flexibility of being followed up by the same specialist smoking advisor.

This paper will inform a wider study being undertaken by Public Health Wales (PHW) which is part of the all Wales NHS trust and are responsible for the health of the people in Wales, and four Health Boards (HB) in Wales called 'Models for Access to Maternal Smoking cessation Support' (MAMSS). Health Boards are present by geographical areas and they deliver the healthcare services in Wales. The MAMSS project is a 12 months project which aims to increase the uptake of stop smoking services and smoking quit rates amongst pregnant women in Wales. MAMSS was started in response to the high prevalence of smoking during pregnancy in Wales and the low uptake of pregnant women in existing SSS. Further, despite evidencebased guidelines from NICE on stop smoking (NICE, 2010), this is not implemented consistently or systematically across Wales. The overall aim of the MAMSS project is to "evaluate the extent to which improvements in the delivery of stop smoking services to pregnant women can increase the proportion of engaged smokers and reduce the number of women smoking during pregnancy" (Public Health Wales, 2013). The Stop Smoking interventions are delivered in the homes of the pregnant women by specialist midwives, maternity support workers and dedicated pregnancy advisors employed by SSW. These are to deliver a service much 
flexible than the 'usual care' delivered by SSW. The 'usual care' service does not provide a home visit option but delivers interventions mainly by group or one-to-one sessions at an allocated venue. This paper will focus on the experiences and views of service users accessing and staff delivering existing stop-smoking services as well as new models of service delivery developed as part of the MAMSS study, with a view to explore how current services can be improved to benefit the pregnant smoker.

\section{LITERATURE REVIEW}

This paper reviews the evidence available on the access and delivery of stop smoking interventions for pregnant women. It focuses on the need for an effective SSS whilst reviewing the referral, engagement and accessibility options available to staff and pregnant women within which training available is considered. In addition, the delivery of specialist stop smoking support is discussed to include ways of providing service holistically, whilst recognising the difficulties encountered in engaging pregnant smokers.

The public health issue of smoking during pregnancy is one that continuously poses problems both for public health professionals and Welsh Government. Methods of reducing current prevalence are being frequently sought to reduce the associated harm caused by smoking in pregnancy. Several projects and research such as Setting Universal Cessation Counselling Education and Screening Standards (SUCCESS), Community Action on Tobacco for Children's Health (CATCH), and BREATHE (Albrecht et al, 2011; McGowan et al, 2010; Bryce et al, 2009) have been carried out to monitor the effectiveness of current services; and to find the most effective way to reduce the prevalence of smoking in pregnancy either by educating or engaging with young girls, younger \& older women and current pregnant smokers. Health education and Promotion Avenue needs to be reviewed and refined consistently to ensure that all young girls and women are aware of the dangers of smoking in pregnancy and of the support available. Hence, it becomes imperative that services provided to support pregnant smokers are easily accessible with a mode of delivery that is flexible and appropriate to maximise the opportunities created to be in contact with, and engage pregnant women who smoke (Albrecht et al, 2011; McGowan et al, 2010).

A Cochrane review by Lumley et al (2009) suggests that continued smoking into late pregnancy can be reduced through the promotion of stop smoking interventions. Thus, effective evidence based SSS is required to increase the uptake of service by pregnant women and bring about an eventual eradication of the public health problem of smoking in pregnancy (Herberts \& Sykes, 2012).

This paper reviews the literature by addressing the referral process, engagement and accessibility available to staff and service users, whilst considering the delivery of specialist stop smoking support to pregnant women. The use of carbon monoxide monitor will be indicated together with the pregnant women's choice of wanting to cut down rather than give up smoking.

\section{A. Brief Intervention Training}

Brief intervention (BI) refers to a non-confrontational way of positively discussing smoking and quitting to encourage the thought of giving up smoking and encourage accessing specialist support when a smoker is ready (Stop Smoking Wales, 2013). The most effective way of identifying and referring smokers who are pregnant was not addressed specifically in the literature even though many studies suggested that the appropriate identification and referral of these women is a way of improving access (McGowan et al, 2010). The study by McGowan et al (2010) seem to suggest that pregnant smokers may not voluntarily access SSS themselves but are more likely to respond through opportunistic BI conducted by health professionals. Brief intervention training empowers the health professional providing routine care to approach the issue of smoking in a client centred way (Lancaster \& Fowler, 2008). However, the likelihood of seeking help by those who go on to take up the service after BI at some point during their pregnancy was not explored in the literatures reviewed.

\section{B. Opt-out Referral Pathway}

Opt-out services where all pregnant women are automatically referred to SSS increased the opportunity for health professionals to engage with pregnant women who smoke with a possibility of an increase in service uptake (NICE, 2010; Bauld et al, 2012). However, the decision to take up the service ultimately lies with the woman regardless of opportunities provided to access or deliver stop smoking services (NICE, 2010; Bauld et al, 2012). An optout service was found to be more beneficial overall compared to opt-in services where women were asked about their smoking habit, although there were no conclusions as to the best health professional to refer or provide behavioural support (McGowan et al, 2010; Lumley et al, 2009). Some authors have suggested that using providers who already engage with supporting women is necessary (Borland et al, 2013). Whilst other literatures found that some providers such as midwives have found breeching the subject and offering support may put a 'strain' on the relationship between the health provider and pregnant woman (Bull, 2007).

Findings have shown that younger women have reported feeling isolated and would benefit from peer support (Radley et al, 2013). It is therefore essential for some women to have peer support as part of the opt-out system, this is especially so for younger women still living at home who have been relatively unsuccessful at giving up smoking and may find it easier to relate to someone similar to themselves. More so, this can also be used as an avenue for promoting pregnant smokers who have been successful not only at accessing and receiving stop smoking intervention but at giving up smoking whilst pregnant (Radley et al, 2013). 


\section{Accessibility}

Various studies such as that by Borland et al (2013,) using semi-structured, in-depth interviews with service providers and pregnant women, seemed to view poor service uptake purely as a problem of accessibility. Poor service uptake was attributed to accessibility and engagement issues, and inconsistent provider practice. To reinforce this, other studies have shown that remoteness of location and human resource shortages can also affect whether targeted women are able to access services, or whether sufficient support is being provided for the delivery of services (Borland et al, 2013). Okolie et al (2010) found that health professionals in rural areas are less likely to want to engage a pregnant woman about her smoking habit. This could be as a result of rural areas consisting mainly of close-knit communities, leaving the health professionals reluctant to introduce any topic which may negatively affect the health professional-pregnant woman relationship already in existence. This could mean addressing a training need for health professionals as there is a strong perception that midwives are very cautious and protective of maintaining a good relationship with pregnant women (Herberts \& Sykes, 2012). Moreover, there was the recognition that although contact with women was required, the women still needed to be encouraged to take up the service (Ruggiero et al, 2003).

Another study using open ended questions found that barriers to accessing services were mainly due to lack of childcare, lack of time and work commitments but found that mothers who spoke more about the benefits of giving up smoking were more likely to access SSS (Ussher et al, 2006). This gives an understanding that whilst tackling barriers to accessing SSS such as advocating home visits, there should also be a 'stronger' message on the benefits of smoking cessation in pregnancy and at the same time the application of caution to avoid frightening pregnant smokers from accessing SSS (Bull, 2007).

\section{DISCUSSION}

Specialist stop smoking support

$>$ Mode of delivery

Two modes of contact between the health provider and the patient for service delivery were identified from current literature as telephone and face to face contact (Ferguson et al, 2012; Baha \& Le Faou, 2009). The use of motivational telephone interviewing to deliver stop smoking support was not always found to be useful (Ferguson et al, 2012; Baha \& Le Faou, 2009), this may be because pregnant smokers find it easier to connect with staff during face-to-face contact when receiving support for such a sensitive issue. Interventions such as the BREATHE intervention using telephone support to deliver SSS to pregnant women was based on evidence from non-pregnant smokers who did not have the added pressure of pregnancy which is one of the reasons that have been given for smoking in pregnancy (Baha \& Le Faou, 2009).

\section{$>$ Targeting stress factors}

The current trend of tackling smoking cessation in pregnancy seems to focus on a holistic approach because stress from multiple sources appears to be a major hindrance to cessation. (Bull, 2007). Sources of stress include psychosocial factors relating to stigma, lack of social support and socio-economic pressure, it is therefore essential that methods of addressing these issues are considered when planning and delivering a SSS (Okolie et al, 2010; Bull, 2007; McGowan et al, 2010).

However, health professionals engaging with pregnant women felt better motivated to support pregnant smokers once they felt the women were ready to give up smoking and a multidisciplinary team was available to support the women's individual needs (Bull, 2007). There is an overall agreement that women who do not take up stop smoking service offers are generally not interested in giving up smoking (McGowan et al, 2010; Baha \& Le Faou, 2009; Ussher et al, 2006; Borland et al, 2013) and are therefore less likely to access services available. Midwives and health visitors feel that the success of delivering SSS once accessed by pregnant smokers lie in the support received from evidence-based training, and professional support from senior colleagues relevant to pregnant smokers as well as family-friendly policies (Bull, 2007; Okolie et al, 2010).

\section{Socio-economic Class}

An unequal rate of access to SSS was found across social-economic classes with women from lower social classes more likely to smoke and not use to SSS (Lowry et al, 2004; McGowan et al, 2010). Ruggiero et al (2003) found that even with intensive recruitment methods coupled with incentives, 384 of 958 (40\%) eligible pregnant smokers from a low-income group refused to enrol in a smoking cessation programme.

In general, women from more affluent areas are more likely to successfully engage with SSS than those from deprived areas (Radley et al, 2013). Less affluent women are four times more likely to smoke just before pregnancy, twice as likely to be pregnant smokers and have higher possibilities of reverting back to smoking after having their babies (Bauld et al, 2012).

Because there is a certain demography of women who are more likely to be pregnant smokers such as those with mental health problems, teenagers and those of low income group; service uptake might improve if these women were targeted and services increased in areas with higher population of such demographics (Borland et al, 2013). This however might raise questions about inequitable service provision so that any such decisions will have to be carefully considered whilst factoring in issues of determinants of health, health needs assessments and cost effectiveness analysis. (Tappin et al, 2010; Radley et al, 2013). 


\section{$>$ Incentives}

NICE (2010) has suggested that the effect of using incentives is encouraging and even in the absence of concrete effectiveness evidence, the idea of incentivising stop smoking services is largely promoted by recent studies (Mantzari et al, 2012; Radley et al, 2013). However, incentives may encourage engagement, but they do not guarantee compliance or quit rates as indicated in the result of the study by Radley et al (2013) which idealises SSS and incentive schemes as being mutually exclusive, that is unable to occur together. Moreover, studies like the comparative qualitative study by Mantzari et al (2012) found that motivation to stop smoking was the same in all pregnant women regardless of incentives. Incentives were more of a bonus and not the main reason for trying to give up smoking in pregnancy.

Overall, regardless of incentives young women carrying their first pregnancy feel more pressure to give up smoking than other mothers or those who have already been pregnant before. This might be as a result of struggling to transition into motherhood (Herberts \& Sykes, 2012).

\section{$>$ Difficulties with engagement}

Some women do not attend stop smoking service appointments even after referral by a health professional; this is because services provided are viewed as an absolute stop smoking when they only feel ready to reduce the number of cigarettes. Some women already struggling with trying to give up other substances such as alcohol, cannabis and other drugs will be unlikely to take up SSS even if a 'perfect' model of access and delivery is made available (Baha \& Le Faou, 2009). They may either agree to an intervention with for example their midwife due to social pressure or agree but lose motivation once back in an unsupportive home environment.

It is pertinent to bear in mind that a 'stronger' public health message of the benefits of stop smoking in pregnancy may not necessarily have as much impact as suggested by Bull (2007), especially with the study methodology used by studies such as that by Ussher et al (2006), which carried out internet based survey using two 10-questions questionnaires. An assumption was made that all who participated in filling the internet questionnaires were pregnant smokers, introducing response bias into their study. This had the potential to influence results of the analysis. Albeit, the study by Baha \& Le Faou (2009) found that women not attending their stop smoking appointments after referral were likely to be in denial of the dangers of smoking which might support the relevance of 'stronger' public health messages on the benefits of smoking cessation in pregnant women. Moreover, Fendall et al (2012) reported the need for a prescriptive intervention after pregnant women during a focus group reported wanting to be told precisely the possible health outcome for themselves and the baby if they did not give up smoking.

\section{$>$ Carbon Monoxide Testing}

Societal pressure not to smoke in pregnancy can inhibit pregnant smokers from admitting to smoking, making it difficult to seek stop smoking service support. One way of determining one's smoking status is by measuring the amount of expired carbon monoxide (CO) in their breath. It is a quick and non-invasive method as the result is known immediately (NICE, 2010). Midwives have been recommended by NICE to perform this test on all pregnant women at their first maternity appointment and on subsequent appointments for those who smoke (NICE, 2010). NICE recommends an expired CO level of 7 PPM or above as the cut-off for referral, however, there appears to be no universally agreed cut-off level in the literature so that women were being referred to SSS with varying measured levels. This is problematic as the amount of expired CO may be affected by passive smoking, exposure to traffic fumes or gases from leaky appliances leading to falsely positive results and wrongs assumptions and potentially wrong accusations by the service deliverer which then leads to a strain in the relationship with the client (Baha \& Le Faou, 2009; McGowan et al, 2010). Bauld et al (2012) noted the problem with the varying levels of expired $\mathrm{CO}$ used for referrals to SSS and suggested using 4PPM as a cut-off for pregnant women instead of the 7PPM advocated by NICE and $\mathrm{CO}$ monitor manufacturers. This lack of standardised protocol also impinges on the delivery of smoking cessation services as noted in the study by Borland et al (2013) where the problem of pregnant women not always admitting to being smokers as a result of stigma was highlighted. It can therefore be inferred that some services do not routinely screen all pregnant women for $\mathrm{CO}$. The use of $\mathrm{CO}$ testing in practise has been mentioned by various studies on its use in most parts of the UK, with NICE (2010) recommending the use of a low cut-off point to avoid missing any pregnant smoker needing help.

\section{CONCLUSION}

This paper has successfully explored the questions of the knowledge, experiences and views of pregnant service users accessing and staff delivering SSS to pregnant women in Wales. Although some of the limitations have been recognised, both the service users and some staff reported that a specialist stop smoking service for pregnant women was deemed most appropriate for supporting pregnant smokers than a non-specialist smoking cessation service, especially with the availability of home visits as part of service provided.

However, not all the midwives in this study acknowledged smoking cessation support as being part of their role, this was explained to be as a result of lack of appropriate training and time required due to other commitments.

Carbon monoxide monitors were yet to be routinely used in practise by all midwives. Its potential and that of having specialists who are midwives, at influencing pregnant women who smoke to accept smoking cessation will need further research with a larger population sample. 
Motivation was closely linked with being pregnant and having an appropriate support system. Moreover, pregnant smokers were more likely to be from deprived areas and generally prefer to cut down than give up smoking completely. Stress was reported as a major factor in the inability to give up smoking, with monetary incentives not necessarily contributing to the motivation to give up. Several barriers to smoking cessation were found and reported within this paper with deprivation, a smoking background, personal perception of stigma, the possibility of attending group stop smoking sessions and lack of flexibility reported as some of the main reason for poor service uptake.

To conclude, members of staff were seen to understand their roles and were aware of training requirements to provide a better service. Although advisors recognised the barriers to service imposed by their job role because they were not specialist, majority of the barriers to service were characteristic of the usual care provided by SSW advisors. Both staff and pregnant women acknowledged that accessibility and flexibility of service were key determinants of service delivery and service uptake, with motivation and having a wider support network being integral to the success of service uptake and successful quitting. Overall, it is unlikely that the problem of smoking in pregnancy will come to an end with the intervention of specialised services only. However, wider strategies can be directed at young school-aged girls to promote a non-smoking mind-set from a young age by introducing health programmes into schools for girls across Wales, as well as the use of peer support by all pregnant smokers to further promote and support smoking cessation amongst this group of women. It should however be acknowledged that pregnant smokers needed to have a specialised service in order to provide a tailored stop smoking service capable of improving service uptake and quit rates.

\section{RECOMMENDATION}

Training about supporting pregnant women that smoke should be provided and appropriately tailored to all health professionals involved in caring for pregnant women. This includes brief intervention training for health professionals in general and specialist training for those providing smoking cessation supports, to include job shadowing experience for new stop smoking advisors.

All midwives should practise the use of $\mathrm{CO}$ monitoring in accordance with NICE guidance, and NICE should provide guidance on the cut-off level of expired $\mathrm{CO}$ which is appropriate for referral into stop smoking services.

\section{REFERENCES}

[1]. Albrecht, S., Kelly-Thomas, K., Osborne, J.W. \& Ogbagaber, S. (2011) The SUCCESS Program for Smoking Cessation for Pregnant Women. Journal of Obstetric, Gynecologic \& Neonatal Nursing. 40 (5), pp. 520-531.
[2]. Baha, M.Y. \& Le Faou, A.L. (2009) Attitude towards cessation among French pregnant smokers: Explaining the poor uptake of specialised support. European Journal of Obstetrics \& Gynecology and Reproductive Biology. 147 (1), pp. 46-51.

[3]. Bauld, L., Hackshaw, L., Ferguson, J., Coleman, T., Taylore, G. \& Salway, R. (2012) Implementation of routine biochemical validation and an 'opt out' referral pathway for smoking cessation in pregnancy. Addiction 107(2), pp. 53-60.

[4]. Bauld, L. \& Coleman, T. (2009) The Effectiveness of Smoking Cessation Interventions during Pregnancy: A Briefing Paper. National Institute for Health and Clinical Excellence.

[5]. Borland, T., Babayan, A., Irfan, S. \& Schwartz, R. (2013) Exploring the adequacy of smoking cessation support for pregnant and postpartum women. BioMed Central Public Health. 13:472.

[6]. Bryce, A., Butler, C., Gnich, W., Sheehy, C. \& Tappin, D.M. (2009) CATCH: development of a home-based midwifery intervention to support young pregnant smokers to quit. Midwifery. 25 (5), pp. 473482.

[7]. Bull, L. (2007) Smoking cessation intervention with pregnant women and new parents (part 2): A focus group study of health visitors and midwives working in the UK. Journal of Neonatal Nursing. 13 (5), pp. 179-185.

[8]. Fendall, L., Griffith, W., Iliff A., Lee, A. \& Radford, J. (2012) Integrating a Clinical Model of Smoking Cessation into Antenatal Care. British Journal of Midwifery. 20 (4), pp. 236-243.

[9]. Ferguson, J. Docherty, G. Bauld, L. Lewis, S. Lorgelly, P. Boyd, K. McEwen, A \& Coleman, T. (2012) Effect of offering different levels of support and free nicotine replacement therapy via an English national telephone quit line: randomised controlled trial. British Medical Journal. 344:e1696.

[10]. Herberts, C \& Sykes, C. (2012) Midwives' Perceptions of Providing Stop-Smoking Advice and Pregnant Smokers' Perceptions of Stop-Smoking Services Within the Same Deprived Area of London. Journal of Midwifery \& Women's Health. 57(1), pp. 67-73.

[11]. James, J. (2007) All Wales Smoking Cessation Service: Annual report 1 April 2006- 31 March 2007. National Public Health service for Wales.

[12]. Lancaster, T \& Fowler, G. (2008) Training health professionals in smoking cessation: Review. Cochrane Library. Issue 4.

[13]. Lowry, R .J. Hardy, S. Jordan, C \& Wayman, G. (2004) Using social marketing to increase recruitment of pregnant smokers to smoking cessation service: a success story. Journal of the royal institute of Public Health. 118 (4), pp. 239-243.

[14]. Lumley, J. Chamberlain, C. Dowswell, T. Oliver, S. Oakley, L \& Watson L (2009) Interventions for promoting smoking cessation during pregnancy. Cochrane Database of Systematic Reviews. Issue 3. 
[15]. McGowan, A. Hamilton, S. Barnett, D. Nsofor, M. Proudfoot, J \& Tappin, D. (2010) 'Breathe': the stop smoking service for pregnant women in Glasgow. Midwifery. 26 (3), pp. e1-e13.

[16]. Mantzari , E. Vogt, F \& Marteau, T. (2012) The effectiveness of financial incentives for smoking cessation during pregnancy: is it from being paid or from the extra aid? BioMed Central Pregnancy and Childbirth. 12:24.

[17]. National Health Service Wales (2006) Health in Wales: Smoking [online].

[18]. National Institute for Health and Care Excellence (2006) Brief interventions and referral for smoking cessation. Public health guidance (PH1).

[19]. National Institute for Health and Care Excellence (2010) Quitting smoking in pregnancy and following childbirth. NICE public health guidance 26 .

[20]. National Health Service Information Centre (2011) Infant Feeding Survey: Early results [online].

[21]. National Institute for Health and Care Excellence (2012) Specifying services for quitting smoking in pregnancy and following childbirth: Contacting women who have been referred and offering initial and ongoing support from specialist advisers.

[22]. National Institute for Health and Care Excellence (2013) Tobacco: Harm reduction approaches to smoking. NICE public health guidance 45.

[23]. O’Callaghan, F. V. Al Mamun, A. O’Callaghan, M. Alati, R. Najman, J. M \& Williams, G.M.(2009) Maternal smoking during pregnancy predicts nicotine disorder (dependence or withdrawal) in young adultsa birth cohort study. The Australian and New Zealand Journal of Public Health. 33 (4), pp. 371- 377.

[24]. Okoli, C. Greaves, L. Bottorf, J \& Marcellus, L. (2010) Health Care Providers' Engagement in Smoking Cessation With Pregnant Smokers. Journal of Obstetric, Gynecologic, \& Neonatal Nursing. 39 (1), pp. 64-77.

[25]. Public Wales (2011) How are current and future population health needs likely to impact on the use of maternity, neonatal, gynaecology and paediatric services in North Wales? - A population profile. http://howis.wales.nhs.uk/sitesplus/888/news/39884 . In Jones S, Tyler E, Brassey J, De Souza S, Reilly R, Paranjothy S (2012) Smoking in Pregnancy: Briefing Paper Reproductive and Early Years Pathfinder Project. Public health Wales.

[26]. Public Health Wales (2012) Stop Smoking Wales [online].

[27]. Public Health Wales (2013) Models for Access for Maternal Smoking Cessation (MAMMS) [online].

[28]. Radley, A. Ballard, P. Eadie, D. MacAskill, S. Donnelly, L \& Tappin, D. (2013) Give It Up For Baby: outcomes and factors influencing uptake of a pilot smoking cessation incentive scheme for pregnant women. Bio-Med Central Public Health . 13:343.

[29]. Ruggiero, L .Webster, K. Jeffrey F. Peipert, J.F \& Wood, C. (2003) Identification and recruitment of low-income pregnant smokers: Who are we missing? Addictive Behaviors. 28 (8), pp.1497-1505.
[30]. Stop Smoking Wales (2013) Brief Intervention Training [online].

[31]. Tappin D.M, MacAskill S, Bauld L, Eadie D, Shipton D, Galbraith L (2010) Smoking prevalence and smoking cessation services for pregnant women in Scotland. Substance Abuse Treatment, Prevention and Policy. 5:1.

[32]. Ussher, M. Etter J \& West R. (2006) Perceived barriers to and benefits of attending a stop smoking course during pregnancy. Patient Education and Counseling. 61 (3), pp. 467-472. 